Proyecciones Journal of Mathematics

Vol. 38, No 1, pp. 49-57, March 2019.

Universidad Católica del Norte

Antofagasta - Chile

\title{
On nearly Lindelöf spaces via generalized topology
}

\author{
Bishwambhar Roy * \\ Women's Christian College, India \\ Received : April 2017. Accepted : November 2018
}

\begin{abstract}
In this paper a new class of sets termed as $\omega_{\mu}$-regular open sets has been introduced and some of its properties are studied. We have introduced $\mu$-nearly Lindelöfness in $\mu$-spaces. We have shown that under certain conditions a $\mu$-Lindelöf space [7] is equivalent to a $\mu$-nearly Lindelöf space. Some properties of such spaces and some characterizations of such spaces in terms of $\omega_{\mu}$-regular open sets are given.
\end{abstract}

2000 AMS subject code : 54A05, 54D20.

Key Words : $\mu$-open set, $\mu$-open set, $\omega_{\mu}$-regular open set, $\mu$-nearly Lindelöf space.

${ }^{*}$ The author acknowledges the financial support from Department of Higher education, Science and technology and Biotechnology, Government of West Bengal, India 


\section{Introduction}

For the last one decade or so, a new area of study has emerged and has been rapidly growing. The area is concerned with the investigations of generalized topological spaces and several classes of generalized types of open sets. The notion of $\omega$-open set in a topological space was introduced by Hdeib [4] which forms a topology finer than the original topology. Our aim here is to study the notion of $\mu$-nearly Lindelöf spaces by using the concept of generalized topology introduced by Á. Császár [2]. We first recall some definitions given in [2]. Let $X$ be a non-empty set and $\exp X$ denote the power set of $X$. We call a class $\mu \subseteq \exp X$ a generalized topology (briefly, GT) [1, 2], if $\varnothing \in \mu$ and $\mu$ is closed under arbitrary union. A set $X$, with a GT $\mu$ on it is said to be a generalized topological space (briefly, GTS) and is denoted by $(X, \mu)$. For a $\operatorname{GTS}(X, \mu)$, the elements of $\mu$ are called $\mu$-open sets and the complements of $\mu$-open sets are called $\mu$-closed sets. A GTS $(X, \mu)$ is called a $\mu$-space [5] or a strong GTS [2] if $X \in \mu$. A $\operatorname{GTS}(X, \mu)$ is called a QTS if $\mu$ is closed under finite intersection. It is easy to observe that $i_{\mu}$ and $c_{\mu}$ are idempotent and monotonic. It is also well known from $[1,2]$ that if $\mu$ is a GT on $X$ and $A \subseteq X, x \in X$, then

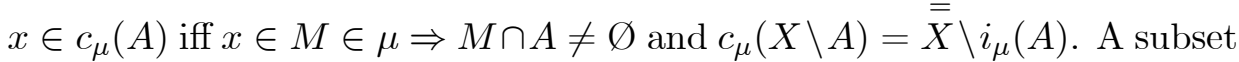
$A$ of a $\operatorname{GTS}(X, \mu)$ is called $\mu r$-open if $A=i_{\mu}\left(c_{\mu}(A)\right)$. The complement of a $\mu$-open set is called a $\mu r$-closed set(see [3] for details).

The purpose of this paper is to study the concept of $\mu$-nearly Lindelöfness in a $\mu$-space. We have also investigated several characterizations of such spaces. In the sequel we have studied some special types of functions which preserve $\mu$-nearly Lindelöfness where $X \in \mu$.

\section{2. $\omega_{\mu}$-regular open sets and its properties}

Definition 2.1. Let $(X, \mu)$ be a GTS. A subset $A$ of $X$ is called an $\omega_{\mu}$ regular open (resp. $\omega_{\mu}$-open [6]) set if for each $x \in A$, there exists a $\mu r$-open ( $\mu$-open) set $U$ containing $x$ such that $U \backslash A$ is countable. The complement of an $\omega_{\mu}$-regular open (resp. $\omega_{\mu}$-open ) set is known as an $\omega_{\mu}$-regular closed (resp. $\omega_{\mu}$-closed $[6]$ ) set.

It follows from Definition 2.1 that every $\omega_{\mu}$-regular open set is an $\omega_{\mu}$ open set and every $\mu r$-open set is an $\omega_{\mu}$-regular open set but the converses are false as shown in Example 2.3. 
Remark 2.2. Let $\mu$ be a GT on a set $X$. Then the following relations hold:

$$
\mu r \text {-open set } \Rightarrow \omega_{\mu} \text {-regular open set } \Rightarrow \omega_{\mu} \text {-open set }
$$

Example 2.3. (a) Let $\Re$ be the set of reals and $\mu=\{\varnothing, Q, I, \Re\}$, where $Q$ is the set of rationals and $I$ is the set of irrationals. Then $(\Re, \mu)$ is a GTS. It can be checked easily that $I \cup\{0\}$ is an $\omega_{\mu}$-regular open set but not a $\mu r$-open set.

(b) Let $X=\Re$ be the set of reals and $\mu=\{A \subseteq X: 0 \in A\} \cup\{\varnothing\}$. Then $\mu$ is a GT on $X$. It can be checked easily that $[0,1)$ is an $\omega_{\mu}$-open set but not an $\omega_{\mu}$-regular open set.

The family of all $\omega_{\mu}$-regular open sets of a $\operatorname{GTS}(X, \mu)$ is denoted by $\omega_{\mu}-R O(X)$.

Proposition 2.4. In a $\operatorname{GTS}(X, \mu), \omega_{\mu}-R O(X)$ is a $\mathrm{GT}$ on $X$.

Proof. It is obvious that $\varnothing$ is an $\omega_{\mu}$-regular open set. Let $\left\{A_{\alpha}: \alpha \in \Lambda\right\}$ be a collection of $\omega_{\mu}$-regular open subsets of $X$. Then for each $x \in \cup\left\{A_{\alpha}: \alpha \in\right.$ $\Lambda\}, x \in A_{\alpha}$ for some $\alpha \in \Lambda$. Thus there exists a $\mu r$-open set $U$ containing $x$ such that $U \backslash A_{\alpha}$ is countable. Now as $U \backslash\left(\cup\left\{A_{\alpha}: \alpha \in \Lambda\right\}\right) \subseteq U \backslash A_{\alpha}$, so $U \backslash\left(\cup\left\{A_{\alpha}: \alpha \in \Lambda\right\}\right)$ is also countable. Thus $\cup\left\{A_{\alpha}: \alpha \in \Lambda\right\}$ is an $\omega_{\mu}^{-}$ regular open set.

Theorem 2.5. A subset $A$ of a $\operatorname{GTS}(X, \mu)$ is an $\omega_{\mu}$-regular open set if and only if for each $x \in A$, there exists a $\mu r$-open set $U_{x}$ containing $x$ and a countable subset $C$ such that $U_{x} \backslash C \subseteq A$.

Proof. Let $A$ be an $\omega_{\mu}$-regular open set in $X$ and $x \in A$. Then there exists a $\mu r$-open set $U_{x}$ containing $x$ such that $U_{x} \backslash A$ is countable. Let $C=U_{x} \backslash A=U_{x} \cap(X \backslash A)$. Then $U_{x} \backslash C \subseteq A$.

Conversely, let $x \in A$ and there exist a $\mu r$-open set $U_{x}$ containing $x$ and a countable subset $C$ such that $U_{x} \backslash C \subseteq A$. Thus $U_{x} \backslash A \subseteq C$ and hence $U_{x} \backslash A$ is a countable set. Thus $A$ is an $\omega_{\mu}$-regular open set in $X$.

Theorem 2.6. Let $(X, \mu)$ be a GTS and $C \subseteq X$. If $C$ is $\omega_{\mu}$-regular closed, then $C \subseteq K \cup B$ for some $\mu r$-closed set $K$ and a countable subset $B$. 
Proof. If $C$ be $\omega_{\mu}$-regular closed, then $X \backslash C$ is $\omega_{\mu}$-regular open and hence for each $x \in X \backslash C$, there exist a $\mu r$-open set $U$ containing $x$ and a countable subset $B$ such that $U \backslash B \subseteq(X \backslash C)$. Thus $C \subseteq X \backslash(U \backslash B)=$ $X \backslash(U \cap(X \backslash B))=(X \backslash U) \cup B$. Let $K=X \backslash U$. Then $K$ is $\mu r$-closed such that $C \subseteq K \cup B$.

\section{3. $\mu$-nearly Lindelöf spaces}

Definition 3.1. A $\mu$-space $(X, \mu)$ is said to be $\mu$-nearly Lindelöf if every cover of $X$ by $\mu r$-open sets has a countable subcover.

A subset $A$ of a $\mu$-space $(X, \mu)$ is said to be $\mu$-nearly Lindelöf relative to $X$ if every cover of $A$ by $\mu r$-open sets of $X$ has a countable subcover of A.

Theorem 3.2. A $\mu$-space $(X, \mu)$ is $\mu$-nearly Lindelöf if and only if every $\omega_{\mu}$-regular open cover of $X$ has a countable subcover.

Proof. Necessity. Let $\left\{U_{\alpha}: \alpha \in \Lambda\right\}$ be any $\omega_{\mu}$-regular open cover of $X$. Then for each $x \in X$, there exists $\alpha(x) \in \Lambda$ such that $x \in U_{\alpha(x)}$. As $U_{\alpha(x)}$ is $\omega_{\mu}$-regular open, there exists a $\mu r$-open set $V_{\alpha(x)}$ containing $x$ such that $V_{\alpha(x)} \backslash U_{\alpha(x)}$ is also countable. Then the family $\left\{V_{\alpha(x)}: x \in X\right\}$ is a cover of $X$ by $\mu r$-open subsets of $X$. Since $(X, \mu)$ is $\mu$-nearly Lindelöf, there exist $\left\{x_{i}: i<\omega\right\} \subseteq X$ such that $X=\cup\left\{V_{\alpha\left(x_{i}\right)}: i<\omega\right\}$. Thus $X=\cup\left[\left\{V_{\alpha\left(x_{i}\right)} \backslash U_{\alpha\left(x_{i}\right)}: i<\omega\right\}\right] \cup\left[\cup\left(U_{\alpha\left(x_{i}\right)}: i<\omega\right)\right]$. For each $\alpha\left(x_{i}\right)$, $V_{\alpha\left(x_{i}\right)} \backslash U_{\alpha\left(x_{i}\right)}$ is a countable set and there exists a countable subset $\Lambda_{\alpha\left(x_{i}\right)}$ of $\Lambda$ such that $V_{\alpha\left(x_{i}\right)} \backslash U_{\alpha\left(x_{i}\right)} \subseteq\left\{U_{\alpha}: \alpha \in \Lambda_{\alpha\left(x_{i}\right)}\right\}$. Thus $X \subseteq\left[\cup\left(\cup\left\{U_{\alpha}: \alpha \in\right.\right.\right.$ $\left.\left.\left.\Lambda_{\alpha\left(x_{i}\right)} ; i<\omega\right\}\right)\right] \cup\left[\cup\left(U_{\alpha\left(x_{i}\right)}: i<\omega\right)\right]$.

Since every $\mu r$-open set is $\omega_{\mu}$-regular open, the sufficiency is obvious.

Theorem 3.3. A $\mu$-space $(X, \mu)$ is $\mu$-nearly Lindelöf if and only if for every family $\left\{F_{\alpha}: \alpha \in \Lambda\right\}$ of $\omega_{\mu}$-regular closed sets with countable intersection property, $\cap\left\{F_{\alpha}: \alpha \in \Lambda\right\} \neq \varnothing$.

Proof. Suppose that $(X, \mu)$ be a $\mu$-nearly Lindelöf space and suppose that $\left\{F_{\alpha}: \alpha \in \Lambda\right\}$ be a family of $\omega_{\mu}$-regular closed subsets of $X$ with countable 
intersection property such that $\cap\left\{F_{\alpha}: \alpha \in \Lambda\right\}=\varnothing$. Let us consider the $\omega_{\mu}$-regular open sets $U_{\alpha}=X \backslash F_{\alpha}$. Then the family $\left\{U_{\alpha}: \alpha \in \Lambda\right\}$ is an $\omega_{\mu}$-regular open cover of $X$. Since $X$ is $\mu$-nearly Lindelöf, there exists a countable subcover $\left\{U_{\alpha_{i}}: \alpha_{i} \in \mathbf{N}\right\}$ such that $X=\cup\left\{U_{\alpha_{i}}: \alpha_{i} \in \mathbf{N}\right\}=$ $\cup\left\{X \backslash F_{\alpha_{i}}: \alpha_{i} \in \mathbf{N}\right\}=X \backslash \cap\left\{F_{\alpha_{i}}: \alpha_{i} \in \mathbf{N}\right\}$. Hence $\cap\left\{F_{\alpha_{i}}: \alpha_{i} \in \mathbf{N}\right\}=\varnothing$. Thus if the family $\left\{F_{\alpha}: \alpha \in \Lambda\right\}$ of $\omega_{\mu}$-regular closed sets be with the countable intersection property, then $\cap\left\{F_{\alpha}: \alpha \in \Lambda\right\} \neq \varnothing$.

Conversely, let $\left\{U_{\alpha}: \alpha \in \Lambda\right\}$ be a cover of $X$ by $\omega_{\mu}$-regular open sets and that for every family $\left\{F_{\alpha}: \alpha \in \Lambda\right\}$ of $\omega_{\mu}$-regular closed sets with countable intersection property, $\cap\left\{F_{\alpha}: \alpha \in \Lambda\right\} \neq \varnothing$. Then $X=\cup\left\{U_{\alpha}: \alpha \in \Lambda\right\}$. Therefore $\varnothing=\cap\left\{X \backslash U_{\alpha}: \alpha \in \Lambda\right\}$ and the family $\left\{X \backslash U_{\alpha}: \alpha \in \Lambda\right\}$ is a family of $\omega_{\mu}$-regular closed sets with an empty intersection. Thus there exists a countable collection $\left\{X \backslash U_{\alpha_{i}}: i \in \mathbf{N}\right\}$ such that $\cap\left\{X \backslash U_{\alpha_{i}}: i \in \mathbf{N}\right\}=\varnothing$. Hence $X=\cup\left\{U_{\alpha_{i}}: i \in \mathbf{N}\right\}$. Thus $X$ is $\mu$-nearly Lindelöf.

Theorem 3.4. Every $\omega_{\mu}$-regular closed subset of a $\mu$-nearly Lindelöf space $(X, \mu)$ is $\mu$-nearly Lindelöf relative to $X$.

Proof. Let $A$ be an $\omega_{\mu}$-regular closed subset of $X$. Let $\left\{U_{\alpha}: \alpha \in \Lambda\right\}$ be a cover of $A$ by $\mu r$-open subsets of $X$. Then for each $x \in X \backslash A$, there exists a $\mu r$-open set $V_{x}$ such that $V_{x} \cap A$ is countable. Since $X$ is $\mu$-nearly Lindelöf and the collection $\left\{U_{\alpha}: \alpha \in \Lambda\right\} \cup\left\{V_{x}: x \in X \backslash A\right\}$ is a cover of $X$ by $\mu r$ open sets, there exists a countable subcover $\left\{U_{\alpha_{i}}: i \in \mathbf{N}\right\} \cup\left\{V_{x_{i}}: i \in \mathbf{N}\right\}$. Since $\cup\left\{V_{x_{i}} \cap A: i \in \mathbf{N}\right\}$ is countable, so for each $x_{j} \in \cup\left\{V_{x_{i}} \cap A: i \in \mathbf{N}\right\}$, there is $U_{\alpha_{x_{j}}} \in\left\{U_{\alpha}: \alpha \in \Lambda\right\}$ such that $x_{j} \in U_{\alpha_{x_{j}}}$ and $j \in \mathbf{N}$. Hence $\left\{U_{\alpha_{i}}: i \in \mathbf{N}\right\} \cup\left\{U_{\alpha_{x_{j}}}: j \in \mathbf{N}\right\}$ is a countable subcover of $\left\{U_{\alpha}: \alpha \in \Lambda\right\}$ and it covers $A$. Therefore $A$ is $\mu$-nearly Lindelöf relative to $X$.

Definition 3.5. A GTS $(X, \mu)$ is said to be $\omega_{\mu}$-regular if for each $x \in X$ and each $\mu$-open set $G_{x}$ in $X$ containing $x$, there exists an $\omega_{\mu}$-regular open set $H_{x}$ such that $x \in H_{x} \subseteq c_{\mu}\left(H_{x}\right) \subseteq G_{x}$.

Definition 3.6. A GTS $(X, \mu)$ is said to be weakly $\omega_{\mu}$-regular if for each $x \in X$ and each $\mu$-open set $U_{x}$ containing $x$ there exists an $\omega_{\mu}$-regular open set $H_{x}$ such that $x \in H_{x} \subseteq U_{x}$.

We note from Definitions 3.5 and 3.6 that every $\omega_{\mu}$-regular space is weakly $\omega_{\mu}$-regular.

Definition 3.7. A $\mu$-space $(X, \mu)$ is said to be $\mu$-Lindelöf [7] if every cover 
of $X$ by $\mu$-open sets has a countable subcover.

A subset $A$ of a $\mu$-space $(X, \mu)$ is said to be $\mu$-Lindelöf relative to $X[7]$ if every cover of $A$ by $\mu$-open sets of $X$ has a countable subcover of $A$.

Theorem 3.8. A weakly $\omega_{\mu}$-regular space is $\mu$-nearly Lindelöf if and only if it is $\mu$-Lindelöf.

Proof. Let $X$ be a weakly $\omega_{\mu}$-regular and $\mu$-nearly Lindelöf $\mu$-space and $\mathcal{U}=\left\{U_{\alpha}: \alpha \in \Lambda\right\}$ be a $\mu$-open cover of $X$. Then for each $x \in X$, there exists $\alpha(x) \in \Lambda$ such that $x \in U_{\alpha(x)}$. Since $X$ is weakly $\omega_{\mu}$-regular, there exists an $\omega_{\mu}$-regular open set $H_{\alpha(x)}$ such that $x \in H_{\alpha(x)} \subseteq U_{\alpha(x)}$. Then $\left\{H_{\alpha(x)}: x \in X\right\}$ is an $\omega_{\mu}$-regular open cover of $X$. Thus by Theorem 3.2, there exists a countable subcover $\left\{H_{\alpha_{x_{i}}}: i \in \mathbf{N}\right\}$. Hence $X$ is a $\mu$-Lindelöf space. The converse part is obvious.

Example 3.9. Let $\Re$ be the set of real numbers and $\mu=\{A \subseteq \Re: 0 \in$ $A\} \cup\{\varnothing\}$. Then $(\Re, \mu)$ is a GTS. It is easy to see that $(\Re, \mu)$ is $\mu$-nearly Lindelöf but not $\mu$-Lindelöf as $\{\{0, x\}: x \in \Re\}$ is a cover of $\Re$ by $\mu$-open sets having no countable subcover.

Corollary 3.10. If $(X, \mu)$ is $\omega_{\mu}$-regular and $\mu$-nearly Lindelöf, then it is $\mu$-Lindelöf.

Definition 3.11. A GTS $(X, \mu)$ is said to be almost $\omega_{\mu}$-regular if for each $x \in X$ and each $\omega_{\mu}$-regular closed set $F$ disjoint from $\{x\}$, there exist disjoint $\mu$-open sets $U$ and $V$ in $X$ such that $F \subseteq U$ and $x \in V$.

Example 3.12. (a) Let $X=\{a, b, c\}$ and $\mu=\{\varnothing,\{a\},\{b\},\{a, b\}\}$. Then $(X, \mu)$ is a GTS. It can be checked easily that $(X, \mu)$ is weakly $\omega_{\mu}$-regular but neither $\omega_{\mu}$-regular nor almost $\omega_{\mu}$-regular.

(b) Let $X=\{a, b, c\}$ and $\mu=\{\varnothing,\{c\},\{a, b\}, X\}$. Then $(X, \mu)$ is a GTS. It can be checked easily that $(X, \mu)$ is $\omega_{\mu}$-regular but not almost $\omega_{\mu}$-regular.

Theorem 3.13. A GTS $(X, \mu)$ is almost $\omega_{\mu}$-regular if and only if for each $x \in X$ and each $\omega_{\mu}$-regular open set $U_{x}$ containing $x$, there exists a $\mu r$-open set $V_{x}$ in $X$ such that $x \in V_{x} \subseteq c_{\mu}\left(V_{x}\right) \subseteq U_{x}$.

Proof. Necessity : Let $U$ be an $\omega_{\mu}$-regular open set with $x \in U$. Then $x \notin X \backslash U$, where $X \backslash U$ is $\omega_{\mu}$-regular closed. Thus there exist disjoint 
$G, H \in \mu$ such that $x \in G, X \backslash U \subseteq H$. So, $x \in i_{\mu}\left(c_{\mu}(G)\right)=V_{x}$ (say), $X \backslash U \subseteq i_{\mu}\left(c_{\mu}(H)\right)=U_{x}$ (say). Thus $x \in V_{x} \subseteq c_{\mu}\left(V_{x}\right) \subseteq U\left(\right.$ as $\left.U_{x} \cap V_{x}=\varnothing\right)$.

The sufficient part is obvious from the hypothesis.

Theorem 3.14. Let $(X, \mu)$ be an almost $\omega_{\mu}$-regular, $\mu$-nearly Lindelöf QTS. Then for every disjoint $\omega_{\mu}$-regular closed sets $C_{1}$ and $C_{2}$, there exist two disjoint $\mu$-open sets $U$ and $V$ such that $C_{1} \subseteq U$ and $C_{2} \subseteq V$.

Proof. Since $(X, \mu)$ is an almost $\omega_{\mu}$-regular space, for each $x \in C_{1}$ there exists a $\mu r$-open set $U_{x}$ containing $x$ such that $c_{\mu}\left(U_{x}\right) \cap C_{2}=\varnothing$ (by Theorem 3.13). Then the family $\left\{U_{x}: x \in C_{1}\right\} \cup\left\{X \backslash C_{1}\right\}$ is an $\omega_{\mu}$-regular open cover of $X$. Since $X$ is $\mu$-nearly Lindelöf, there exist $\left\{x_{i}: i<\omega\right\} \subseteq X$ such that $X=\left[\cup\left\{U_{x_{i}}: i<\omega\right\}\right] \cup\left(X \backslash C_{1}\right)$. Thus $C_{1} \subseteq \cup\left\{U_{x_{i}}: i<\omega\right\}$ and $c_{\mu}\left(U_{x_{i}}\right) \cap C_{2}=\varnothing$. Similarly, there exist a family of $\mu r$-open subset $V_{y_{i}}$ with $\left\{y_{i}: i<\omega\right\} \subseteq X$ such that $C_{2} \subseteq \cup\left\{V_{y_{i}}: i<\omega\right\}$ and $c_{\mu}\left(V_{y_{i}}\right) \cap C_{1}=\varnothing$. Let $G_{k}=U_{x_{k}} \backslash \cup\left\{c_{\mu}\left(V_{y_{i}}\right): i=1,2, \ldots, k\right\}$ and $H_{k}=V_{y_{k}} \backslash \cup\left\{c_{\mu}\left(U_{x_{i}}\right): i=\right.$ $1,2, \ldots, k\}$ and $U=\cup\left\{G_{i}: i<\omega\right\}$ and $V=\cup\left\{H_{i}: i<\omega\right\}$. Then $U$ and $V$ are two disjoint $\mu$-open sets containing $C_{1}$ and $C_{2}$ respectively.

Definition 3.15. A function $f:(X, \mu) \rightarrow(Y, \lambda)$ is said to be $\delta_{\omega_{\mu}}{ }^{-}$ continuous if for each $\lambda$-regular open set $V$ of $Y$ containing $f(x)$, there exists a $\omega_{\mu}$-regular open set $U$ of $X$ containing $x$ such that $f(U) \subseteq V$.

Theorem 3.16. Let $f:(X, \mu) \rightarrow(Y, \lambda)$ be a surjective $\delta_{\omega_{\mu}}$-continuous function. If $X$ is $\mu$-nearly Lindelöf, then $Y$ is $\lambda$-nearly Lindelöf.

Proof. Let $\mathcal{V}=\left\{V_{\alpha}: \alpha \in \Lambda\right\}$ be a $\lambda r$-open cover of $Y$. Then for each $x \in X$, there exists $V_{\alpha(x)} \in \mathcal{V}$ such that $f(x) \in V_{\alpha(x)}$. Thus there exists an $\omega_{\mu}$-regular open set $U_{\alpha(x)}$ of $X$ containing $x$ with $f\left(U_{\alpha}(x)\right) \subseteq V_{\alpha}(x)$. So $\left\{U_{\alpha}(x): x \in X\right\}$ is a cover of $X$. Hence there exists $\left\{x_{i}: i<\omega\right\} \subseteq X$ such that $X=\cup\left\{U_{\alpha\left(x_{i}\right)}: i<\omega\right\}$. Thus $Y=f(X)=f\left(\cup\left\{U_{\alpha}\left(x_{i}\right): i<\omega\right\}\right)=$ $\cup\left\{f\left(U_{\alpha\left(x_{i}\right)}\right): i<\omega\right\} \subseteq \cup\left\{V_{\alpha\left(x_{i}\right)}: i<\omega\right\}$.

Definition 3.17. A function $f:(X, \mu) \rightarrow(Y, \lambda)$ is said to be $\omega_{\mu} R$ continuous if $f^{-1}(V)$ is $\omega_{\mu}$-regular open in $X$ for each $\lambda$-open set $V$ of $Y$. 
Theorem 3.18. Let $f:(X, \mu) \rightarrow(Y, \lambda)$ be a surjective $\omega_{\mu} R$-continuous function. If $X$ is $\mu$-nearly Lindelöf, then $Y$ is $\lambda$ - Lindelöf.

Proof. Let $\left\{V_{\alpha}: \alpha \in I\right\}$ be a cover of $Y$ by $\lambda$-open subsets of $Y$. Then $\left\{f^{-1}\left(V_{\alpha}\right): \alpha \in I\right\}$ is a cover of $X$ by $\omega_{\mu}$-regular open sets in $X$. Thus there exists a countable subset $I_{0}$ of $I$ such that $X=\cup\left\{f^{-1}\left(V_{\alpha}\right): \alpha \in I_{0}\right\}$. Thus $Y=f(X)=\cup\left\{V_{\alpha}: \alpha \in I_{0}\right\}$ showing $Y$ to be $\lambda$-Lindelöf.

Definition 3.19. A function $f:(X, \mu) \rightarrow(Y, \lambda)$ is said to be $\omega_{\mu} R$-closed if for each $\mu r$-closed set $F$ of $X, f(F)$ is $\omega_{\lambda}$-regular closed in $Y$.

Definition 3.20. A GTS $(X, \mu)$ is said to be a $P_{r}^{*}$-space if every countable union of $\mu r$-open sets is a $\mu r$-open set.

Theorem 3.21. Let $(X, \mu)$ be a $P_{r}^{*}$-space and $f:(X, \mu) \rightarrow(Y, \lambda)$ be an $\omega_{\mu} R$-closed surjection such that for each $y \in Y, f^{-1}(y)$ is $\mu$-nearly Lindelöf relative to $X$. If $(Y, \lambda)$ is $\lambda$-nearly Lindelöf then $(X, \mu)$ is $\mu$-nearly Lindelöf.

Proof. Let $\left\{U_{\alpha}: \alpha \in \Lambda\right\}$ be a $\mu r$-open cover of $X$. For each $y \in Y, f^{-1}(y)$ is $\mu$-nearly Lindelöf relative to $X$, so there exists a countable subset $\Lambda(y)$ of $\Lambda$ such that $f^{-1}(y) \subseteq \cup\left\{U_{\alpha}: \alpha \in \Lambda(y)\right\}$. Let $U(y)=\cup\left\{U_{\alpha}: \alpha \in \Lambda(y)\right\}$ and $V(y)=Y \backslash f(X \backslash \bar{U}(y))$. Then $V(y)$ is $\omega_{\lambda}$-regular open in $Y$ containing $y$ such that $f^{-1}(V(y)) \subseteq U(y)$ (as $f$ is $\omega_{\mu} R$-closed). Since $\{V(y): y \in Y\}$ is an $\omega_{\lambda}$-regular open cover of $Y$, there exist $\left\{y_{i}: i<\omega\right\} \subseteq Y$ such that $Y=\cup\left\{V_{y_{k}}: k<\omega\right\}$. Therefore $X=f^{-1}(Y)=\cup\left\{f^{-1}\left(V_{y_{k}}\right): k<\omega\right\} \subseteq$ $\cup\left\{U_{y_{k}}: k<\omega\right\}=\cup\left\{U_{\alpha}: \alpha \in \Lambda\left(y_{k}\right), k<\omega\right\}$. Thus $(X, \mu)$ is $\mu$-nearly Lindelöf.

Acknowledgement : The author is thankful to the referee for his valuable comments for the improvement of the paper.

\section{References}

[1] Á. Császár, Generalized topology, generalized continuity, Acta Math. Hungar., 96, pp. 351-357, (2002). 
[2] Á. Császár, Generalized open sets in generalized topologies, Acta Math. Hungar., 106, pp. 53-66, (2005).

[3] Á. Császár, $\delta$ - and $\theta$ - modifications of generalized topologies, Acta math. Hungar., 120, pp. 275-279, (2008).

[4] H. Z. Hdeib, w-continuous functions, Dirasat Jour., 16 (2), pp. 136-153, (1989).

[5] T. Noiri, Unified characterizations for modifications of $R_{0}$ and $R_{1}$ topological spaces, Rend. Circ. Mat. Palermo, 5 (2), pp. 29-42, (2006).

[6] B. Roy, More on $\mu$-Lindelöf spaces in $\mu$-spaces, Questions and Answers in Gen. Topol., 33, pp. 25-31, (2015).

[7] M. S. Sarsak, On $\mu$-compact sets in $\mu$-spaces, Questions and Answers in Gen. Topol., 31 (1), pp. 49-57, (2013).

\author{
Bishwambhar Roy \\ Department of Mathematics \\ Women's Christian College \\ 6, Greek Church Row \\ Kolkata-700 026 \\ India \\ e-mail : bishwambhar_roy@yahoo.co.in
}

